\title{
Subject Index Vol. 7, 1997
}

Acetazolamide 201,235

Acute stroke management 113

Anticoagulants 100

Anticoagulation 239

Antiphospholipid antibodies 100

Aspiration 220

Aspirin 100

Asterixis 175

Atherosclerosis 338

Austria 163

B mode ultrasonography

338 Barthel Index 22 Basilar infarction 94 Binswanger's encephalopathy 324 Blood pressure 205,264

- $\quad$ - variability 214

Body temperature 64

Brain edema 349

Brainstem 175

Cardiac myxoma 239

Cardioembolic stroke 28 Carotid arteries 138,232

- $\quad$ artery disease 201

- $\quad$ atherosclerosis 232

- endarterectomy 34,201

- stenosis 138,280 Carotid-cavernous fistula

273 Case fatality 154 Centrum ovale infarction

138 Cerebral aneurysm 57,226

- arteriovenous malformation 57

- artery occlusion 57,226

- $\quad$ - stenosis 57

- blood volume 235

- circulation 14

- hemorrhage 284

- $\quad$ infarction 220,232, 284,318

- isch(a)emia 94,284

- vasomotor reserve 332

Cerebrovascular disease

264

- $\quad$ disorders 70,168,175, 214,258

- $\quad$ event 48 
Characterization 289

China 89

Cholesterol 10

Orcadian blood pressure patterns 338

Circle of Willis 57

Clinical trials 94

Coagulation 100

Coil embolization 226

Collaboration 205

Color Doppler flow imaging 289

Complications 220

Consensus 289

Contralateral hemispheric hypometabolism 43

Contrast-enhanced ultrasound 57

Cortical infarct 245

Ggggofillness 258

Costs and cost analysis 163

Dalteparin 28

Decision analysis 34

Deep infarct 245

Dementia 14,48,359

Diabetes 144

Disability 19,22

Discharge disposition 168

Dural arteriovenous malformation 150

- sinus thrombosis 150

Economics 258

Edema 324

Effectiveness 77

Efficiency 77

Ehlers-Danlos syndrome 273

Electroencephalography 2

Embolic infarction 239

Emergency care organization 113

En do vascul ar treatment 226

Epidemiology 48,154

Erythrocyte aggregation 70, 144

Estonia 154

External carotid artery 338

Extracranial internal carotid artery 289 
Fibrinogen 64,70,144

Fibrinolysis 94

Finapres 214

Force 187

Frontal gait apraxia 359

Functional imaging 187

Geomagnetic activity 345 Glutamate 245 Glycosylated hemoglobin

144

HDL cholesterol 10 Hemorheology 70 Hemorrhage 318 Heparin 318 Hospital discharge statistics)

163 Hospitalization 251 Human brain 235 Hypertension 144 Hyperviscpsi^l 44

Incidence 154 Inter-rater reliability 19 Internal carotid artery 338

- $\quad$ - occlusion 180

Intima media thickness

338 Intracerebral haemorrhage

85 Intracranial hematoma 239

- $\quad$ pressure 349

Isch (a)emic cerebrovascular disease 28

- $\quad$ stroke 10,144, 180

Ischemia 2

Joseph Haydn 359

Lacunar infarction 138 Lacunes 324 LDL cholesterol 10 Logistic regression model

168 Low-molecular-weight

heparin 28 Lupus anticoagulant 100

Magnetic resonance

imaging 235 Microsurgical embolectomy

226 Midbrain 175 
Middle cerebral artery infarct 43

Misdiagnosis 284

Monitoring 113

Motor cortex physiology 187

Movement disorder 175

MR imaging and MR angiography 289

MRI 239

Neurointensive care 113 Neurological risks 289 Neuroprotection 245 Non-insulin-dependent diabetes mellitus 332

Outcome 85,205 - assessment 258 Overlap syndromes 2

Patent foramen ovale 100 Pathological studies 289 Pathophysiology 2 Plasminogen activator 318 Plasticity 187 Platelet aggregation 100 Positron emission tomography 43,187 Power Doppler 57 Prediction 85 Predictors 168 Prognosis 19,22,48,264 Progressing stroke 64 Projected trends 163 Pulsatile tinnitus 150

Quality of health care, Netherlands 251

Randomised controlled

trial 205 Recovery 19 Red blood cell aggregation

70 Regional cerebral blood

flow 187 Rehabilitation 168,187 Reorganization 187 Retinopathy 332 Russia 345

Seizures 2

Small deep borderzone infarct 280 
Solar activity 345

Spiral computed tomographic angiography 289

Splenectomy 180

StrokeEp4,48,89,154, 187,245,284,345,349

- $\quad$ assessment 251

- incidence 163

- $\quad$ intensive care 113

- mechanism 144

- outcome 100

- progression 28

unit(s) 77,284 Subcortical vascular encephalopathy 359

Systemic review 205

Therapy 94 Thrombocytosis 180 Thrombolysis 94 Timing 89 Tissue type 318 Transcranial Doppler 180

- $\quad$ - sonography 57

- ultrasound 332 Transhemispheric dia-

schisis 43 Transient ischemic attack

28 Treatment, endovascular

273 Tremor 175 Type-HI collagen 273

Ultrasonics 14,201 Urokinase 94

Validity 22 Vascular dementia 48 Vasomotor reactivity 14

201 Vomiting 220

Warfarin 100

X-ray angiography 289

\section{KAIVGER}

E-Mail karger@karger.chFax+41613061234 
C 1997 S. Karger AG, Basel 